\title{
Pneumatose Intestinal e Complicações de Endocardite por Enterococcus faecium: a propósito de um caso clínico
}

\author{
Pneumatosis Intestinalis and Complications \\ of Infective Endocarditis Due to Enterococcus faecium: a case report
}

\author{
Ana Mondragão ${ }^{1}$, Ana Raquel Ramos ${ }^{1}$, Marta Barbedo ${ }^{1}$ \\ ${ }^{1}$ Serviço de Medicina Interna do Centro Hospitalar Gaia e Espinho, Vila Nova de Gaia, Portugal
}

\begin{abstract}
Resumo
Os Enterococos pertencem à flora comensal humana e têm sido reconhecidos como importantes agentes causadores de infeções. As infeções causadas por estes microrganismos têm vindo a aumentar, especialmente entre os idosos, doentes com doenças cardíacas degenerativas e válvulas cardíacas protésicas, bem como em manipulações dos tratos gastrointestinal e urinário. São responsáveis por cerca de 8-17\% das Endocardites Infeciosas, sendo o Enterococos o terceiro principal responsável - 90\% dos casos devido ao Enterococcus faecalis e com menos de 5\% causada por Enterococcus faecium. A morbilidade e mortalidade de endocardite por Enterococos é elevada. A pneumatose intestinal habitualmente é assintomática, com complicações em apenas 3\% dos casos, incluindo obstrução intestinal, perfuração e hemorragia. Uma vez em circulação, os Enterococos têm elevada afinidade para válvulas cardíacas espessadas ou protésicas. As principais complicações cardíacas são os abcessos perivalvulares; raramente surgem outras complicações, nomeadamente perfuração do miocárdio e aneurismas valvulares. A cirurgia está indicada em complicações graves.
\end{abstract}

Palavras-Chave: Endocardite, Enterococos, Enterococcus faecium, Fístula, Pneumatose Intestinal. Palabras Clave: Endocarditis Infecciosa, Enterococcus, Endocarditis, Pneumatosis Intestinal, Enterococcus faecium

\section{Introdução}

A infeção por Enterococos é responsável por 8 a 17\% de todas as endocardites infeciosas (EI) ${ }^{1}$. Apesar dos avanços no diagnóstico e tratamento, a mortalidade da endocardite por Enterococos não diminuiu nas últimas décadas ${ }^{2,4}$. Ainda que variáveis como a idade avançada, insuficiência cardíaca e êmbolos cerebrais têm sido reconhecidas como fatores de risco para elevada mortalidade, estudos multicêntricos têm avaliado o papel de outras variáveis, como a aquisição de infeção nosocomial, a presença de comorbilidades, ou a mudança de suscetibilidade antimicrobiana dos Enterococos como fatores determinantes do prognóstico $0^{2,3,4}$.

Estes microrganismos estão na patogenia de uma importante percentagem de infeções nosocomiais a nível mundial, sendo cada vez mais associados com infeções do trato urinário, endocardites, infeções intra-abdominais e pélvicas, infeções relacionadas com cateteres, infeções de feridas cirúrgicas e infeções do sistema nervoso centra|5,6,11. As duas espécies de Enterococos mais comuns são Enterococcus faecalis e Enterococcus faecium, sendo o primeiro mais comum e responsável por $80-90 \%$ das El por Enterococos ${ }^{7}$. Ambos são capazes de formar biofilmes ${ }^{4,6}$ - complexos de moléculas ligadas irreversivelmente nas várias superfícies bióticas e mecânicas ${ }^{7,8}$. Gradualmente, as infeções contraí-

\begin{abstract}
Enterococci belong to the commensal human flora and have been recognized as important causative agents of infections. The infections caused by these organisms have been increasing, particularly among the elderly, patients with degenerative heart disease and cardiac prosthetic valves, as well as manipulation of the gastrointestinal and urinary tracts. They are responsible for about 8-17\% of infectious endocarditis, being the Enterococci responsible for the main third - 90\% of cases due to Enterococcus faecalis and less than 5\% caused by Enterococcus faecium. The morbidity and mortality of enterococcal endocarditis is high. The pneumatosis intestinalis is usually asymptomatic, and complications are present in only $3 \%$ of cases, including intestinal obstruction, perforation and hemorrhage. Once in circulation, Enterococcus have high affinity for thickened or prosthetic heart valves. The main cardiac complications are the perivalvulares abscesses; rarely arise other complications, including perforation of the myocardium and valvular aneurysms. Surgery is indicated in severe complications.
\end{abstract}

Keywords: Endocarditis, Enterococcus, Enterococcus faecium, Fistula, Pneumatosis Cystoides Intestinalis

das por Enterococos estão associadas a instrumentação dos tratos urinário e gastrointestinal (Gl), bem como em situações de falta de integridade ou trauma da mucosa ${ }^{7,11,12}$.

As complicações cardíacas por El são propensas quando ocorrem enfarte agudo do miocárdio ou bacteriemia, devido à necrose das fibras papilares e do processo inflamatório envolvente ${ }^{4,10,11}$. A complicação mais comum é a formação de abcessos ${ }^{14,15}$. As fístulas intracardíacas podem desenvolver-se devido à extensão da infeção da válvula do miocárdio adjacente ${ }^{14,21}$. Raramente, podem levar à formação de aneurismas, disseção aórtica e perfuração do miocárdio ${ }^{5,14,22}$. Habitualmente, 0 Estafilococos é a espécie mais isolada nas complicações mais graves ${ }^{21,22 ;}$ no entanto, com 0 aumento da prevalência das infeções por Enterococcus, têm surgido relatos de complicações cardíacas por Enterococcus faecium.

A pneumatose intestinal (PI) corresponde a gás no interior da parede intestinal, levando à disrupção da mucosa ${ }^{17,18}$. As complicações ocorrem em cerca de 3\% dos doentes, incluindo obstrução intestinal, vólvulo, perfuração e hemorragia ${ }^{17,19,20}$. Uma vez em circulação, os Enterococos têm elevada afinidade para válvulas cardíacas espessadas ou protésicas ${ }^{18,20}$. 
Figura 1. Regurgitaçaõ aórtica grave $\left(^{\star}\right)$, evidenciando incompetência da válvula aórtica $(->)$ e, consequentemente, insuficiência aórtica grave. VE - ventrículo esquerdo

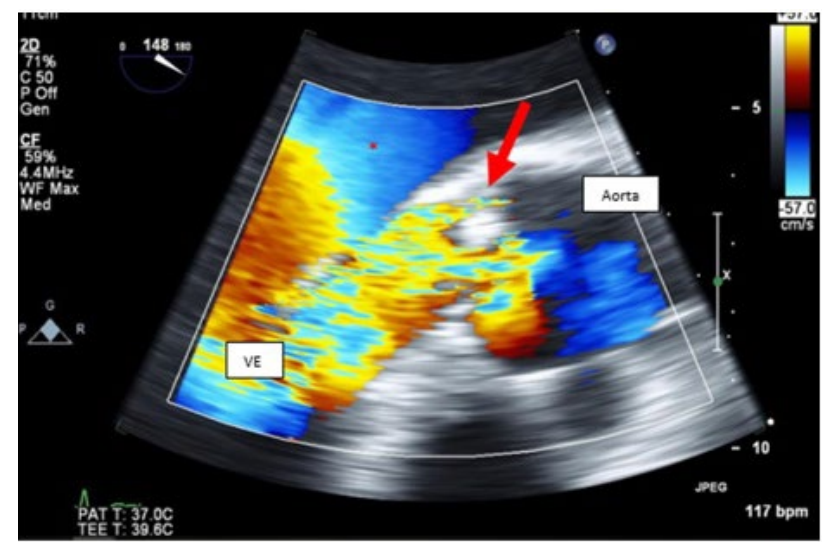

Figura 2. Pseudoaneurisma (->) visível na cúspide coronária direita

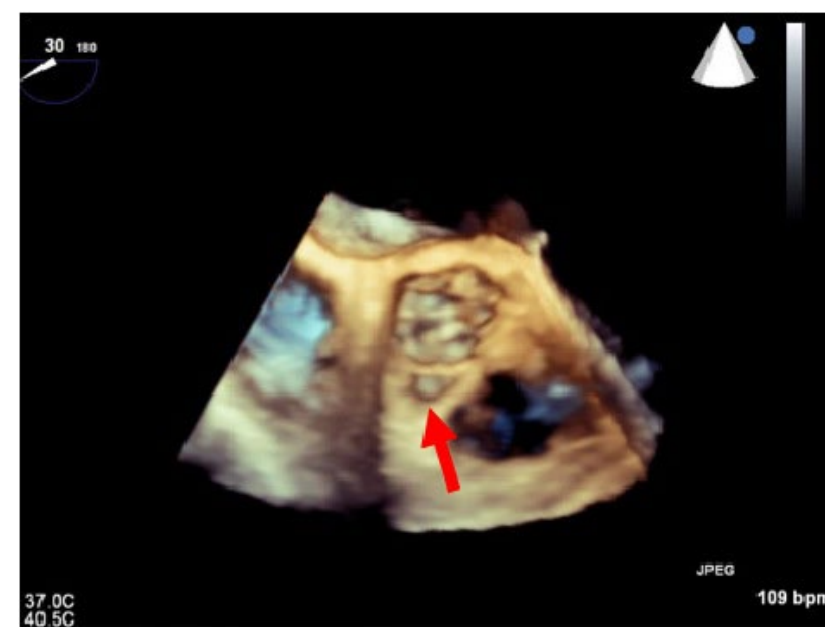

Figura 3. Fluxo regurgitante (->) ente a aorta e o Ventrículo Direito. VE-ventrículo esquerdo; VD ventrículo direito

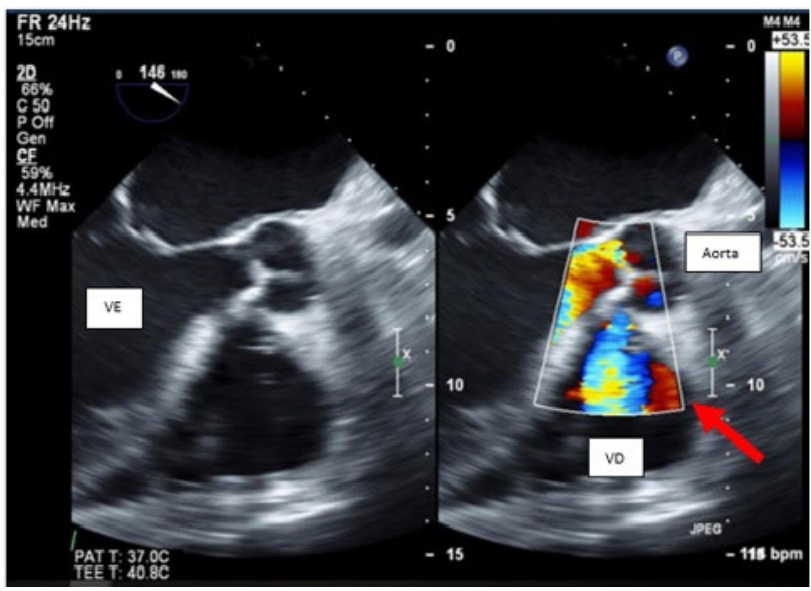

\section{Caso clinico}

Homem de 70 anos, com antecedentes pessoais de Diabetes Mellitus tipo 2, Hipertensão e Dislipidémia, recorreu ao serviço de urgência (SU) por astenia, edemas dos membros inferiores, dor hipocôndrio e região lombar esquerdas. Ao exame objetivo no SU destacou-se discurso lentificado mas coerente, desidratado e descorado, polipneico e com tiragem supraclavicular, Tensão Arterial de 94-44 mmHg, Frequência Cardíaca de 118 bpm, Temperatura axilar de $37,1^{\circ} \mathrm{C}$; abdómen discretamente doloroso à palpação difusa e profunda, sem sinais de irritação peritoneal; edemas dos membros inferiores até à raiz da coxa. Do estudo laboratorial salientou-se hemoglobina de 5,6 g/dL (13.0 - $18.0 \mathrm{~g} / \mathrm{dL})$, creatinina de $3,33 \mathrm{mg} / \mathrm{dL}(0.67-1.17 \mathrm{mg} / \mathrm{dL})$, ureia de $216 \mathrm{~g} / \mathrm{dL}(17$ - $50 \mathrm{mg} / \mathrm{dL}$ ), potássio de $7,19 \mathrm{mmo} / \mathrm{L}$ (3.5 - 5.0), lactatos de 6,1 $\mathrm{mmol} / \mathrm{L}$, INR de 1,64 e D-dímeros 2,54 $\mu \mathrm{g} / \mathrm{ml}(<0.5 \mu \mathrm{g} / \mathrm{ml})$. Durante a permanência no SU apresentou hematoquézias e melenas, em quantidade abundante, motivo pelo qual realizou colonoscopia de urgência, que mostrou divertículos no cólon direito, alguns com restos hemáticos escuros e PI

Realizou endoscopia digestiva alta que descartou hemorragia ativa gástrica. Radiografia torácica sem evidência de imagens de condensação sugestivas de pneumonia ou pneumatocelos. 0 ecocardiograma transtorácico (ETT) excluiu hipocinesias ou dilatação das camaras cardíacas; apresentava dilatação moderada da aurícula esquerda, cúspides da válvula aórtica espessadas e com sinais de fibrose, insuficiência aórtica (IAo) de grau moderado e função globalmente preservada. Transferido para unidade de cuidados intensivos com diagnóstico de choque hipovolémico.

No segundo dia de internamento objetivada febre de $38.0^{\circ} \mathrm{C}$, que indiciou causa sética para além da hipovolémia, como contribuição do choque apresentado. Colheu hemoculturas e iniciou empiricamente piperacilina-tazobactam $4,5 \mathrm{~g}$ de $6 / 6$ horas.

Ao $7^{\circ}$ dia de internamento foi isolado Enterococcus faecium em hemoculturas sensível a ampicilina, tendo iniciado antibioterapia dirigida. Foi requisitado ecocardiograma transesofágico (ETE), que revelou válvula aórtica morfologicamente tricúspide, espessada, com presença de estruturas filiformes móveis, aderentes à cúspide coronária direita e não coronária sugestivas de vegetações; IAo grave (figura 1) com jato regurgitante largo, ocupando quase a totalidade da câmara de saída do ventrículo esquerdo (VE); presença de pseudoaneurisma na dependência da cúspide coronária direita (figura 2); fluxo diastólico a nível da câmara de entrada do ventrículo direito (VD), junto à válvula tricúspide, correspondendo a fistulização entre a aorta e o VD (figura 3).

Foi submetido a cirurgia de substituição da válvula aórtica e correção de fístula.

Gradualmente o doente foi recuperando do quadro infecioso, lesões órgãos-alvo e compensação da insuficiência cardíaca. 0 ecocardiograma de controlo após 4 semanas mostrou prótese biológica em posição aórtica normalmente inserida, regurgitação protésica mínima; visualização do óstio da coronária esquerda, apresentando calibre normal e bom fluxo; compromisso ligeiro da função sistólica ventricular esquerda global.

\section{Discussão}

os Enterococos são parte da flora intestinal normal do ser humano $0^{5,10,11}$. Têm sido reconhecidos como importantes agentes patogénicos sendo responsáveis por $10 \%$ dos casos de El (seguido dos Estreptococos e Estafilococos) 1,3,10,11. A infeção por estes microrganismos têm sido crescentemente 
associados a manipulação dos tratos Gl e urinário e cateteres ${ }^{1,6,12}$.

Aproximadamente 90\% dos casos de endocardite de Enterococos são causadas por Enterococcus faecalis, com menos de 5\% causada por Enterococcus faecium ${ }^{2,14}$. A morbilidade e mortalidade de endocardite por Enterococos é elevada. A percentagem de doentes que necessitam de cirurgia cardíaca é de 42\%5,15,17.

Neste caso clínico a porta de entrada do Enterococcus faecium foi a instrumentação intestinal (colonoscopia) associada à PI que permitiu a disseminação hematogénica do agente, uma vez que a barreira da mucosa intestinal não se encontrava coesa. A maioria dos casos de PI apresenta-se de forma assintomática ${ }^{16,17}$. A sua patogénese não está completamente estabelecida, no entanto, pode ser causada por isquemia do intestino, trauma mecânico, doença intestinal inflamatória / autoimune, neoplasias do intestino, infeção do intestino, imunossupressão induzida por drogas e doença pulmonar obstrutiva crónica ${ }^{16,20}$. Em alguns casos, a PI é um achado incidental, ao passo que, em outros, ela anuncia uma condição intra-abdominal com risco de vida ${ }^{19,20}$. A incidência da PI parece estar a aumentar ${ }^{18}$; novos medicamentos e procedimentos cirúrgicos têm sido relatados como os responsáveis deste aumento ${ }^{18,20}$.

0 doente desenvolveu choque sético e hipovolémico por hemorragia digestiva com várias disfunções orgânicas: neurológica, renal, hematológica, hepática e endócrina.

De notar a importância da suspeita clínica precoce, essencialmente em doentes submetidos a instrumentações ou trauma da mucosa. 0 ecocardiograma transtorácico é 0 exame de primeira linha recomendado na suspeita de endocardite infeciosa. Na maioria dos estudos, a sensibilidade ETT varia entre $40-63 \%$ e a do ETE entre $90-100 \%{ }^{23}$. 0 tamanho da vegetação também afeta a sensibilidade ETT, uma vez que apenas $25 \%$ das vegetações $<5 \mathrm{~mm}$ e $70 \%$ das que têm entre 6-10 mm são identificadas ${ }^{22,23}$. Doença de base de válvula (válvulas esclerótica ou calcificadas), pequenas vegetações $(<2 \mathrm{~mm})$ e obesidade podem influenciar a precisão do diagnóstico de ETT; nestes casos o ETE é essencial para 0 diagnóstico e exclusão de endocardite ${ }^{23,24}$.

As complicações cardíacas mais comuns da El são os abcessos valvulares ${ }^{5,14,21}$. A fistulização intracardíaca (aorto-auricular ou aorto-ventricular) pode ocorrer pela infeção da válvula adjacente ao miocárdio ${ }^{2,21}$ Raramente, ocorrem aneurismas, disseção aórtica, e perfuração do miocárdio5,14,21,22. 0 doente apresentou uma complexidade de consequências da El: fistulização entre a aorta e o VD, insuficiência cardíaca grave e aneurisma valvular.

A intervenção cirúrgica é aplicada em cerca de metade dos doentes com El com complicações graves. Deve ser considerada precocemente na fase ativa, ou seja, enquanto 0 doente está sob antibioterapia, a fim de evitar embolização sistémica, insuficiência cardíaca progressiva e dano estru-

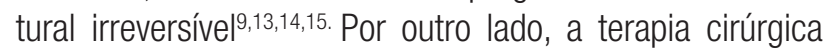
durante a fase ativa da doença está associada a risco signi- ficativo ${ }^{14,15}$. A cirurgia é justificada em doentes com caraterísticas de alto risco que tornam improvável a possibilidade de cura com o tratamento antibiótico e que não tenham comorbilidades impeditivas de cirurgia cardíaca ${ }^{15,16,21,22}$.

A infeção nosocomial é um fator importante de risco de mortalidade por El $\mathrm{E}^{2,4,7}$. Estima-se que 0 risco de contrair endocardite por Enterococos nosocomial possa aumentar dado 0 aumento de incidência de infeções nosocomiais e da prevalência das comorbilidades de risco. Assim, grande ênfase deve ser colocado na prevenção e na deteção precoces. A variabilidade e complexidade clínicas da El ditam que as recomendações e guidelines devem ser usadas para apoiar e não para suplantar decisões na orientação de cada doente individualmente.

\section{Bibliografia}

1. Anders D, Rasmus R, Henning B, Christian H et al. Valvular Heart Disease: Enterococcus Faecalis Infective Endocarditis. Circulation. 2013; 127: 1810-1817.

2. Simone D, Tleyjeh IM, Anavekar N, Baddour LM. Temporal trends in infective endocarditis epidemiology from 2007 to 2013 in Olmsted County. Am Heart J. 2015;170(4):8306.

3. Somarajan SR, La Rosa SL, Singh KV, Roh JH, et all. The fibronectin-binding protein fnm contributes to adherence to extracellular matrix components and virulence of Enterococcus Faecium. Infect Immun. 2015;83(12):4653-61

4. Somarajan SR, La Rosa SL, Singh KV, Roh JH, et al. The fibronectin-binding protein EfbA contributes to pathogenesis and protects against infective endocarditis caused by Enterococcus Faecalis. Infect Immun. 2015; 83(12):4487-94.

5. Higuita N, Huycke M. Enterococcal disease, epidemiology, and implications for Treatment. Enterococci From Commensals to Leading Causes of Drug Resistant Infection 2014.

6. Mohamed JA, Huang DB. Biofilm formation by Enterococci. Journal of Medical Microbiology. 2007;56 (Pt 12):1581-8.

7. Fisher K, Phillips C. The ecology, epidemiology and virulence of Enterococcus. Microbiology 2009, 155: 1749-1757.

8. O'Driscoll T, Crank c, Vancomycin-resistant enterococcal infections: epidemiology, clinical manifestations, and optimal management. Infect Drug Resist. 2015; 8: 217-230.

9. Thuny F, Grisoli D, Cautela H, Riberi A, Raoult D, Habib G. Infective Endocarditis: prevention, diagnosis, and management. Canadien Journal of Cardiology. 2014;30(9):10461057

10. Sydnor E, Perl R. Hospital epidemiology and infection control in acute-care settings. Clin Microbiol Rev. 2011; 24(1):141-173.

11. Fernández-Guerrero ML, Herrero L, Bellver M, Gadea I, Roblas RF, de Górgolas M. Nosocomial Enterococcal endocarditis: a serious hazard for hospitalized patients with enterococcal bacteraemia. J Intern Med. 2002;252(6):510-5.

12. Barros M, Martinelli $R$, Rocha $H$. Enterococcal urinary tract infections in a university hospital: clinical studies. Braz J Infect Dis 2009; 13:4

13. Miro J, Pericas J, Del Rio A. A New Era for Treating Enterococcus Faecalis Endocarditis: Ampicillin plus Short-Course Gentamicin or Ampicillin plus Ceftriaxone; That is the Question!. Circulation 2013; 217: 1763-1766

14. Baddour L, Chair F, Wilson W. et all. Infective Endocarditis in Adults: Diagnosis, Antimicrobial Therapy, and Management of Complications. Circulation 2015; 132: 00-00.

15. Prendergast DM, FCRP, Tornos P. Valvular Heart Disease: Changing Concepts in Disease Management Surgery for Infective Endocarditis - Who and When?. Circulation 2010; 121: 1141-1152.

16. Zhang H, Jun S, Brennan T. Pneumatosis Intestinalis: Not Always a Surgical Indication. Case Reports in Surgery 2012.

17. Berritto D, Crincoli R, lacobellis F, Pizza N et all. Primary Pneumatosis Intestinalis of Small Bowel: A Case of a Rare Disease. Case Reports in Surgery 2014.

18. Ho L, Paulson E, Thompson W. Pneumatosis Intestinalis in the Adult: Benign to Life-Threatening Causes. American Journal of Roentgenology 2007; 188: 6.4

19. Pearl BL. Pneumatosis Intestinalis: a review. Radiology 1998; 207(1):13-9.

20. Mitsuyoshi A, Hamada S, Tachibana T, Momono T. Pathogenic mechanisms of intestinal pneumatosis and portal venous gas: should patients with these conditions be operated immediately?. Surg Case Rep 2015.

21. Stawicki S, Firstenberg M, Lyaker M, Russell S, Evans D. Septic embolism in the intensive care unit. Int J Crit IIIn Inj Sci. 2013; 3(1): 58-63.

22. Saad M, Isbitan A, Roushdy A, Shamoon F. Left Atrial Wall Dissection: A Rare Sequela of Native-Valve Endocarditis. Tex Heart Inst J. 2015;42(2): 178-180.

23. Evangelista A, Gonzalez-Alijas M. Echocardiography in infective endocarditis. Heart. 2004; 90(6): 614-617.

24. Lindner J, Case R, Dent J, Abbott R, Scheld W, Kaul S. Diagnostic Value of Echocardiography in Suspected Endocarditis. Circulation. 1996; 93: 730-736.0 